\title{
BMJ Open Physiotherapy and combined cognitive- behavioural therapy for patients with chronic pelvic pain syndrome: results of a non-randomised controlled feasibility trial
}

\author{
Christian A Brünahl, ${ }^{1,2}$ Susanne G R Klotz (D) , ${ }^{1,3}$ Christoph Dybowski, ${ }^{1}$ \\ Rebecca Albrecht, ${ }^{1}$ Johanna Höink, ${ }^{4}$ Margit Fisch, ${ }^{2}$ Gesche Ketels, ${ }^{3}$ \\ Bernd Löwe (1) ${ }^{1}$
}

To cite: Brünahl $\mathrm{CA}$ Klotz SGR, Dybowski C, et al. Physiotherapy and combined cognitive-behavioural therapy for patients with chronic pelvic pain syndrome: results of a non-randomised controlled feasibility trial. BMJ Open 2021;11:e053421. doi:10.1136/ bmjopen-2021-053421

- Prepublication history and additional supplemental material for this paper are available online. To view these files, please visit the journal online (http://dx.doi.org/10.1136/ bmjopen-2021-053421)

CAB and SGRK contributed equally.

GK and BL contributed equally.

Received 15 May 2021

Accepted 22 November 2021

Check for updates

(C) Author(s) (or their employer(s)) 2021. Re-use permitted under CC BY-NC. No commercial re-use. See rights and permissions. Published by BMJ.

For numbered affiliations see end of article.

Correspondence to Dr Christian A Brünahl; c.bruenahl.ext@uke.de

\section{ABSTRACT}

Objective To explore feasibility in terms of delivering and evaluating a combination of physiotherapy and psychotherapy for patients with chronic pelvic pain syndrome (CPPS).

Design Prospective non-randomised controlled pilot study.

Setting Tertiary care facility with a specialised interdisciplinary outpatient clinic for patients with CPPS. Participants A total of 311 patients was approached; 60 participated. 36 patients were included in the intervention group (mean age \pm SD 48.6 years $\pm 14.8 ; 52.8 \%$ female) and 24 in the control group (mean age \pm SD 50.6 years $\pm 14.5 ; 58.3 \%$ female). Fourteen participants were lost to follow-up.

Interventions Participants were non-randomly allocated to the intervention group with two consecutive treatment modules (physiotherapy and cognitive behavioural therapy) with a duration of 9 weeks each or to the control group (treatment as usual).

Main outcome measures Feasibility was operationalised in terms of delivering and evaluating the therapeutic combination. Regarding eligibility as the first aspect of feasibility, willingness to participate, dropout and satisfaction were assessed; for the second aspect, standardised self-report questionnaires measuring healthrelated quality of life, depression severity and pain were applied.

Results Although eligibility and willingness-to-participate rates were low, satisfaction of the participants in the intervention group was high and dropout rates were low. Results indicated a small and non-significant intervention effect in health-related quality of life and significant effects regarding depression severity and pain.

Conclusions The combination of physiotherapy and psychotherapy for patients with CPPS seems to be feasible and potentially promising with regard to effect. However, a subsequent fully powered randomised controlled trial is needed.

Trial registration number German Clinical

Trials Register (DRKS00009976) and ISRCTN (ISRCTN43221600).

\section{Strengths and limitations of this study}

- A combination of physiotherapy and psychotherapy is recommended for patients with chronic pelvic pain syndrome (CPPS); this therapeutic combination is being investigated in this non-randomised controlled feasibility study.

- The fact that both women and men are affected by CPPS was taken into account by including both genders in this study.

- This study was designed as a feasibility study, so that statements on acceptance, feasibility and evaluation methodology are possible; however, due to insufficient power, no robust statements on the difference between the groups are viable.

- In addition to the feasibility testing, various patientrelevant outcomes, for example, quality of life and pain, were evaluated, which will enable sample size estimation for future, fully powered randomised clinical trials.

- Randomisation could not be carried out, thus the comparability of the two groups is limited.

\section{INTRODUCTION}

Chronic pelvic pain syndrome (CPPS) is a common chronic pain condition with pain perceived in pelvis-related structures and organs without an apparent pathology for at least 6 months. ${ }^{1}$ Worldwide, prevalence rates in the general population range from $4 \%$ to $26.6 \%$ in women ${ }^{23}$ and $2 \%$ to $18 \%$ in men. ${ }^{45}$ Several risk and contributing factors exist, ${ }^{6}$ but the aetiology of CPPS is still unclear. ${ }^{7}$

Several treatment strategies including psychotherapeutic and physiotherapeutic approaches exist, yet for most of these programmes, a distinct benefit was not found.$^{8-11}$ The physiotherapeutic approach with the currently best evidence with respect to pain reduction and improvement in quality 
of life is manual trigger point therapy alone or in combination with active therapy elements. ${ }^{11}$ As for psychotherapy, somatocognitive approaches, which encourage body awareness and reflection on pain cognitions, might be helpful in reducing pain as demonstrated in a randomised controlled trial (RCT). ${ }^{10}$ However, existing reviews demonstrated that the successful treatment of CPPS remains challenging and that single treatment strategies often fail to be satisfactory. ${ }^{9}$ A combination of physiotherapy and psychotherapy might be a promising approach in reducing symptoms and increasing quality of life, ${ }^{10}$ so that a multidisciplinary treatment approach is highly recommended. ${ }^{1812}$ Nonetheless, to the best of our knowledge, no study has tested the combination of physiotherapy and psychotherapy.

Another argument for a combination of treatment modalities is the heterogeneity of symptoms among patients with CPPS. The spectrum includes urogenital, gastroenterological and/or sexual dysfunction. ${ }^{13}$ CPPS is also associated with myofascial ${ }^{12} 14$ and psychopathological symptoms as well as a decreased health-related quality of life. ${ }^{12}{ }^{15-20}$ Furthermore, there seems to be a linkage between myofascial and psychosocial factors. ${ }^{14}$ The aim of this study was to explore the feasibility of combining physiotherapy and psychotherapy in a common therapy approach for female and male patients with CPPS in terms of delivering and evaluating the therapeutic combination.

\section{MATERIAL AND METHODS \\ Study design}

The study was based on the principles of a "cohort multiple RCT proposed by Relton et al. ${ }^{21}$ Participants were recruited from a specialised outpatient clinic for patients with CPPS based at the University Medical Centre Hamburg-Eppendorf. From August 2012 to December 2017, several studies were conducted within the Interdisciplinary Research Platform CPPS. ${ }^{11}{ }^{14-2022-24}$ In the CPPS outpatient clinic, patients underwent multimodal diagnostic algorithm consisting of psychosomatic, physiotherapeutic, urologic and gynaecologic assessments. Patients signed informed consent, which allowed the contact for this study. The protocol for the study was published ${ }^{23}$ (see online supplemental file 1 for the original study protocol)

\section{Patient and public involvement}

Patients or the public were not involved in the design, the reporting or the dissemination plans of this pilot study due to its explorative nature. Patients were involved in the conduct of the trial by participating in one of the study arms. The intervention group was asked to share their experiences including burden and time expenditure associated with the intervention.

\section{Participants}

All potentially eligible patients from the outpatient clinic cohort were contacted. Inclusion criteria included diagnosis of CPPS according to the European Association of Urology (EAU) guidelines ${ }^{1}$ and the International Association for the Study of Pain, ${ }^{25}$ informed consent, age $\geq 18$ years and sufficient German language skills. Exclusion criteria were delusional disorders or substance dependences with the exception of nicotine or painkillers, and acute suicidal tendencies. In addition, patients were not eligible for the intervention group if they had expected absences during the treatment period for more than four therapy units or received ongoing physiotherapeutic or psychotherapeutic treatment; however, participation in the control group was possible. All participants who fulfilled inclusion criteria and signed informed consent were non-randomly allocated to either intervention group or control group. The assignment to the intervention group was based on whether the participant would be able to regularly attend the treatment sessions at the University Medical Centre Hamburg-Eppendorf. The targeted overall size for the intervention group was $\mathrm{n}=36$ and $\mathrm{n}=18$ for the control group.

\section{Intervention group}

A combination of consecutive cognitive behavioural therapy (CBT) and physiotherapy was used in the intervention group. Both therapy modalities were applied in sex homogenous groups in separate modules with a 4-week break between each module. The physiotherapy module was a combination of three 90 min group sessions and six individually scheduled treatment sessions, each lasting $60 \mathrm{~min}$ for 9 weeks. Following the German physiotherapeutic concept of reflective respiratory physiotherapy (Reflektorische Atemtherapie), ${ }^{26}$ the single sessions included heat applications, manual techniques, specific therapeutic movements and educational parts, whereas group sessions focused on active exercises, selfmanagement strategies and education. The psychotherapeutic intervention incorporated 9 weekly 90 min group sessions CBT including theory parts, group discussions and progressive muscle relaxation. ${ }^{27}$ Key topics for the cognitive behavioural intervention were behaviour analysis, positive self-messages, reduction of fear-avoidance-beliefs and behaviour, improvement of physical activity, development of coping strategies, management of catastrophising cognitions and enhancement of social support. A supplementary work book based on the work of Tripp and Nickel ${ }^{28}$ was developed. Participants who had accumulated more than six sessions dropped out of the intervention group.

\section{Control group}

The control group received treatment as usual. The patients were allowed to participate in standard medical care as performed in Germany. This includes, for example, outpatient treatment by a general practitioner or specialist. Hence, they did not receive any specific treatment within this study.

\section{Assessments}

Measurements of all participants were taken at the time of the visit of the outpatient clinic (t1), during the 
recruitment process at baseline (t2) and at the end of the second intervention module (t6). The intervention group was assessed additionally at the beginning (t3) and the end of the first intervention module ( $\mathrm{t} 4)$, at the beginning of the second module (t5), and 4 weeks after the end of the second module $(\mathrm{t} 7)$.

Feasibility of delivering the combined intervention was operationalised in terms of willingness to participate, reasons for refusing to participate and attendance rate. In addition, the acceptance of this therapeutic intervention by the patients was operationalised by a questionnaire assessing the satisfaction of the participants. This questionnaire was designed specifically for this study and contained Likert scales as well as open questions, which gave participants the opportunity to share their thoughts on this combined intervention.

A major concern of this feasibility study was also to provide effect sizes for power calculations for randomised clinical trials to be planned in the future. For this purpose, the effect sizes for different self-report scales were calculated. A power calculation for the present study was consequently not performed, also due to the nature of a feasibility study. The conduct of the inferential statistical analyses, including the determination of effect sizes, also served to analyse the feasibility of the analysis methods for future studies. When interpreting statistical significance in the context of this study, the small sample size, the insufficient power and the non-randomised design must be taken into account. Thus, the main psychometric outcome for the feasibility of the evaluation, the health-related quality of life, was measured with the 12-Item Short-Form Health Survey (SF-12). ${ }^{29}$ Additionally, somatic symptom severity, anxiety severity and depression severity were assessed with the German version ${ }^{30}$ of the Perceived Stress Questionnaire, ${ }^{31}$ the Patient Health Questionnaire (PHQ)-15, ${ }^{32}$ the Generalized Anxiety Disorder Scale ${ }^{33}$ and the PHQ$9,{ }^{34}$ respectively. The German version ${ }^{35}$ of the Chronic Prostatitis Symptom Index of the National Institute of Health (NIH-CPSI) ${ }^{36}$ and an adapted version for women with $\mathrm{CPPS}^{37}$ were used to measure the symptom burden. Pain in conjunction with disability, perception and catastrophising were measured using the German version ${ }^{38}$ of the Pain Disability Index (PDI) ${ }^{39}$ the German version ${ }^{40}$ of the Pain Catastrophizing Scale (PCS) ${ }^{41}$ and the German version ${ }^{42}$ of the Short-Form McGill Pain Questionnaire. ${ }^{43}$ In the physiotherapeutic examination of the intervention group, performed at the time points $\mathrm{t} 3$, $\mathrm{t} 5$, and $\mathrm{t} 7$, tender and trigger points in predefined muscles were manually palpated.

Two adaptations in the outcome measures had to be made after registration: Originally, it was planned to use attainment of individual patient goals in the intervention group measured with the goal attainment scale after each module and 4 weeks after overall treatment. However, the patients were not used to goal setting and the assessment of their goals resulted in feelings of discomfort and insecurity. Hence, goal attainment was dropped as an outcome. The other previously planned outcome, selective attention on pain-related stimuli as measured by a computer-based dot-probe task, was also dropped due to technical difficulties, which arose during the study process.

\section{Statistical analysis}

$\mathrm{X}^{2}$ tests respectively Fisher's exact tests and t-tests for independent groups were calculated for baseline comparisons. Regarding feasibility with emphasis on acceptance, the eligibility rate, the willingness-to-participate rate and the dropout rate were calculated. Additionally, the most frequent reasons for not being eligible, not willing to participate and for dropping-out were presented. Moreover, we compared whether absence differed between modules and whether the overall treatment satisfaction differed from each module by conducting repeated measure analyses of variance.

Prior to the efficacy estimation analysis, which was done in order to gain insight into feasibility of evaluation, missing values in the self-report instruments were imputed using the expectation-maximisation estimation method, ${ }^{44}$ provided that completion rate of a questionnaire for a particular participant at a particular time point was at least $60 \%$. To establish consistency of efficacy estimations, all analyses were adjusted for baseline and sex as well as the interaction between sex and group affiliation at $\mathrm{t} 2$ and $\mathrm{t} 6$. The primary efficacy estimations were defined as the differences between intervention and control group after the treatment ( $\mathrm{t} 6$ ) using analyses of covariance with adjustments for the respective baseline values at t2. Furthermore, potential sequence effects within the intervention group (psychotherapy followed by physiotherapy vs physiotherapy followed by psychotherapy) were analysed by comparing the outcomes at the end of the treatment ( $t 6)$. In addition, sex effects were interpreted comparing the intervention and the control group at the end of the treatment.

Due to the exploratory nature of this study, corrections for multiple testing were not applied. For all efficacy estimations as well as comparisons of the absence and the treatment satisfaction rates, Cohen's $d$ was calculated as an indicator of effect size. The effect sizes were classified as small $(\mathrm{d} \geq 0.2)$, medium $(\mathrm{d} \geq 0.5)$ or large $(\mathrm{d} \geq 0.8){ }^{45}$ Two-tailed $p$ values $<0.05$ were considered significant. All statistical analyses were conducted with IBM SPSS V.24. In addition to the quantitative analyses, the trajectories for measurements of quality of life and CPPS symptoms were presented in line graphs. Furthermore, anecdotal quotes from the free text fields in the questionnaires in German were translated and used to illustrate the range of feedback.

\section{RESULTS}

From October 2012 to June 2017, 311 persons visited the specialised outpatient clinic. Of these, 103 patients did not meet the inclusion criteria or displayed no interest in study participation at the initial screening; thus, 208 


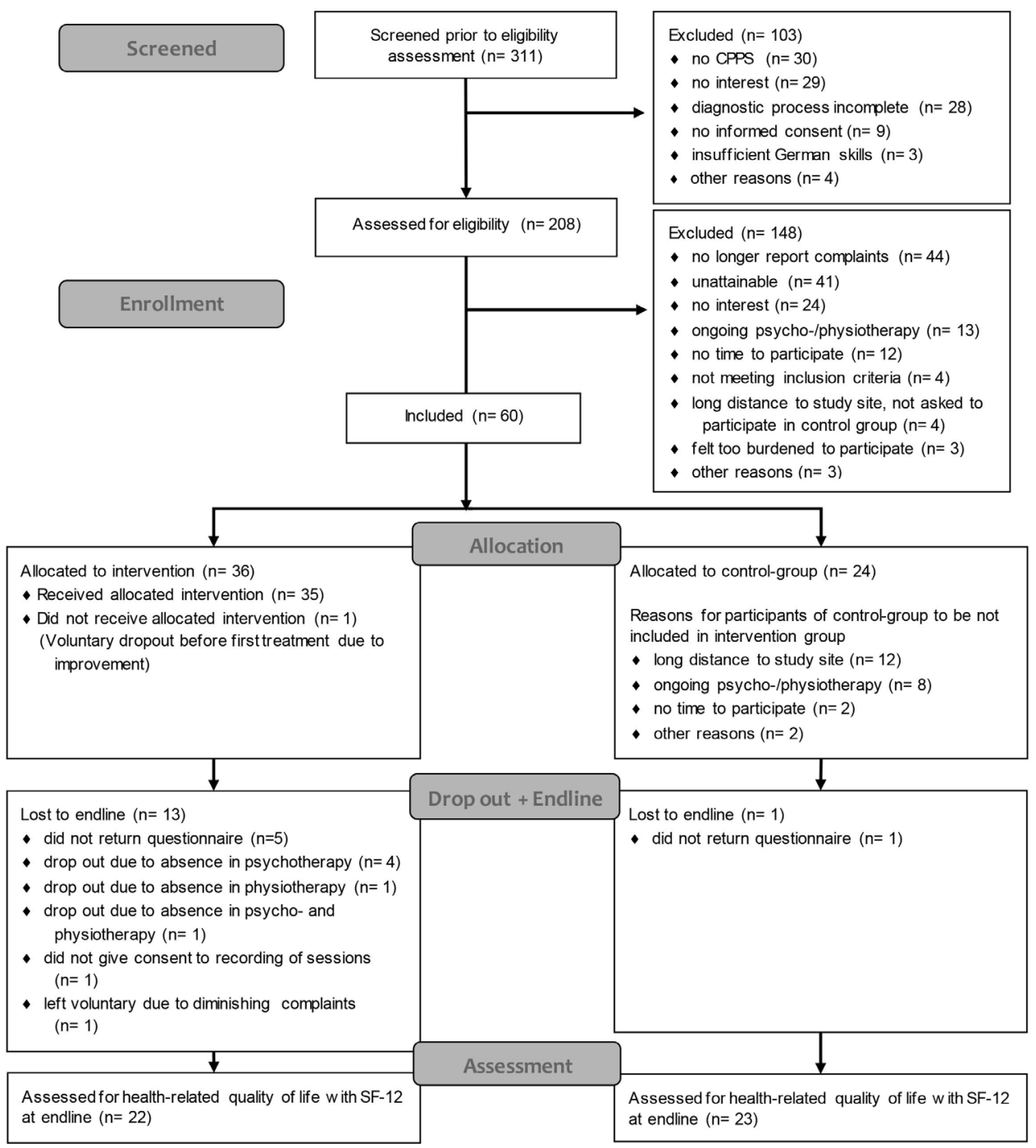

Figure 1 Flow of participants. CPPS, chronic pelvic pain syndrome; SF-12: 12-Item Short-Form Health Survey.

patients were further assessed for eligibility. Of these, an additional 148 patients were excluded due to failure to meet the inclusion criteria or other reasons, with 36 participants remaining in the intervention group and 24 participants remaining in the control group (figure 1). Table 1 illustrates the demographic and psychometric characteristics of the participants. No significant differences between the groups were found.

\section{Feasibility of delivering and satisfaction}

The eligibility rate, when considering all screened persons $(n=311)$, was $44.7 \%$. The main reasons for ineligibility was absence of a CPPS diagnosis and unattainability of patients. Of all eligible persons $(n=172)$, 60 consented to take part in the study; resulting in a willingness-to-participate rate of $34.8 \%$. Patients who were eligible but rejected participation indicated mostly to have no interest or no time. Of the 36 persons in the intervention group, one participant dropped out prior to the first therapy unit and nine participants dropped out during the intervention period-resulting in a dropout rate of $27.8 \%$.
The adjusted average proportion of missed sessions was $\mathrm{M}=36.33 \% \quad(\mathrm{SE}=4.93)$ for the psychotherapeutic module, and $\mathrm{M}=30.03 \%$ ( $\mathrm{SE}=6.24$ ) for the physiotherapeutic module revealing no significant differences.

In general, patients gave high ratings of treatment satisfaction (table 2). The following quotes from the satisfaction questionnaires were selected to illustrate the breadth of patient feedback:

The CPPS study has helped me managing the daily life with my pain and [...] I can get better through the day. Talking about perception of the pain and its treatment $[\ldots]$ has positively affected me.

The manual, the group, and the conversations were helpful. But I still had the need to talk and in the group, I was not confident enough to talk about everything (I would have liked to.).

The interaction with other affected people (patients) was helpful. The contents are easy/good to take into practice. The duration of the group therapy was, 
Table 1 Comparison of demographic and clinical characteristics at baseline

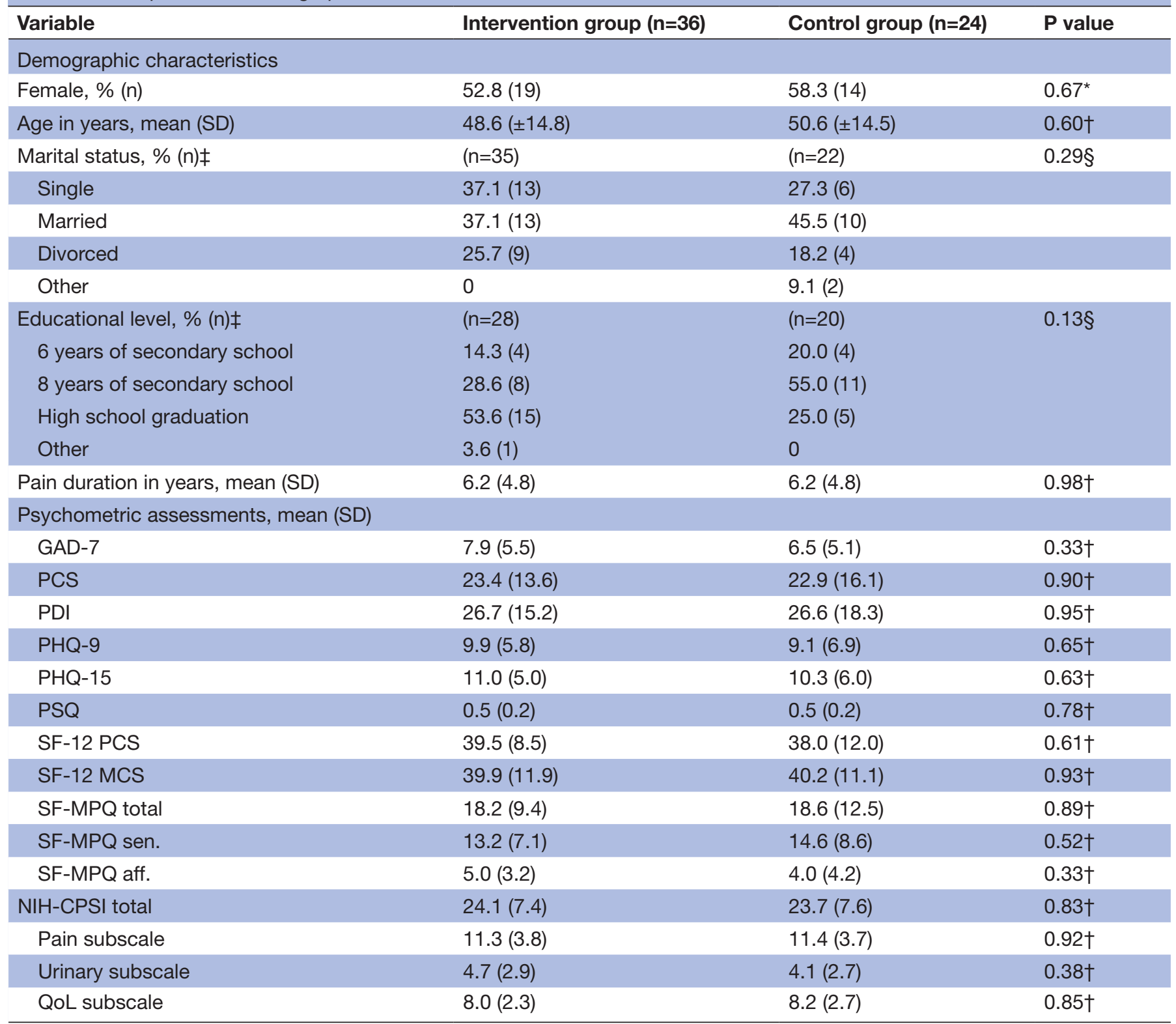

${ }^{\star} \mathrm{X}^{2}$.

†t-test for independent samples.

$\ddagger$ Assessed at outpatient clinic visit (t1).

§Fisher's exact test.

GAD-7, Generalised Anxiety Disorder Screener; NIH-CPSI, Chronic Prostatitis Symptom Index of the National Institutes of Health; PCS, Pain Catastrophizing Scale; PDI, Pain Disability Index; PHQ-9, Patient Health Questionnaire 9 (depressive symptoms); PSQ, Perceived Stress Questionnaire; QoL, Quality of Life; SF-12 MCS, 12-Item Short-Form Health Survey Mental Component Summary; SF-MPQ, Short-Form McGill Pain Questionnaire; SF-MPQ aff., affective subscale of Short-Form McGill Pain Questionnaire; SF-MPQ sen., sensory subscale of Short-Form McGill Pain Questionnaire; SF-12 PCS, 12-Item Short-Form Health Survey Physical Component Summary.

in my opinion, too short. The double number of appointments would be appropriate for the input.

\section{Feasibility of evaluation and estimation of efficacy}

As indicated by the main efficacy estimations, which serve as indicators for feasibility of evaluation, no significant differences or medium effect sizes were found for the SF-12 at the end of the intervention (table 3). With respect to the secondary outcomes, the intervention group reported significantly lower symptom burden as measured by the PDI ( $\mathrm{p}=0.02, \mathrm{~d}=-0.73)$, and the PHQ-9 $(\mathrm{p}=0.04, \mathrm{~d}=-0.62)$. Table 4 displays the results of the analysis of sex-related effects. Neither main effects for sex nor sex*group interaction effects were significant.

Regarding the analysis of sequence effects within the intervention group, no significant differences were found in the SF-12. With respect to the secondary outcomes, the 
Table 2 Treatment satisfaction

\begin{tabular}{|c|c|c|c|c|c|c|c|c|c|}
\hline & \multirow{2}{*}{\multicolumn{2}{|c|}{ All }} & \multirow{2}{*}{\multicolumn{2}{|c|}{ Female }} & \multirow{2}{*}{\multicolumn{2}{|c|}{ Male }} & \multicolumn{3}{|c|}{ Overall comparisons } \\
\hline & & & & & & & \multirow{2}{*}{$\begin{array}{l}\text { Modules* } \\
\text { p (d) }\end{array}$} & \multirow{2}{*}{$\begin{array}{l}\text { Sex } \\
p(d)\end{array}$} & \multirow{2}{*}{$\begin{array}{l}\text { Modulessex* } \\
p(d)\end{array}$} \\
\hline & $\mathbf{N}$ & Est. M (SE) & $\mathbf{N}$ & Est. M (SE) & $\mathbf{N}$ & Est. M (SE) & & & \\
\hline $\begin{array}{l}\text { Psychotherapeutic } \\
\text { module }\end{array}$ & 25 & $5.4(0.3)$ & 14 & $5.1(0.4)$ & 11 & $5.6(0.4)$ & & & \\
\hline
\end{tabular}

Items: 'Would you recommend ...?' ; scale from 1 = 'does not apply at all' to 7 = 'fully applies'; higher values correspond with higher treatment satisfaction.

${ }^{*}$ Overall treatment vs psychotherapeutic module versus physiotherapeutic module.

Est. M, estimated mean.

sequence psychotherapy-physiotherapy was significantly superior to the sequence physiotherapy-psychotherapy in pain reduction as measured by the NIH-CPSI pain subscale $(\mathrm{p}=0.03, \mathrm{~d}=-1.12)$.

Figure 2 displays the courses of the most important outcome variables across all times of measurement. Besides the afore-mentioned results, the figure suggests reductions in the physical and mental component summaries of the SF-12 and increases in the PDI, the NIHCPSI, the PHQ- 9 and the PCS between $t 6$ and follow-up in the intervention group.

\section{DISCUSSION AND CONCLUSIONS}

This study explored feasibility of a combined psychotherapy and physiotherapy in patients with CPPS in terms

Table 3 Post-treatment (t6) comparisons between the intervention group and the control group, adjusted for baseline (t2), sex, and the interaction of sex ${ }^{*}$ group

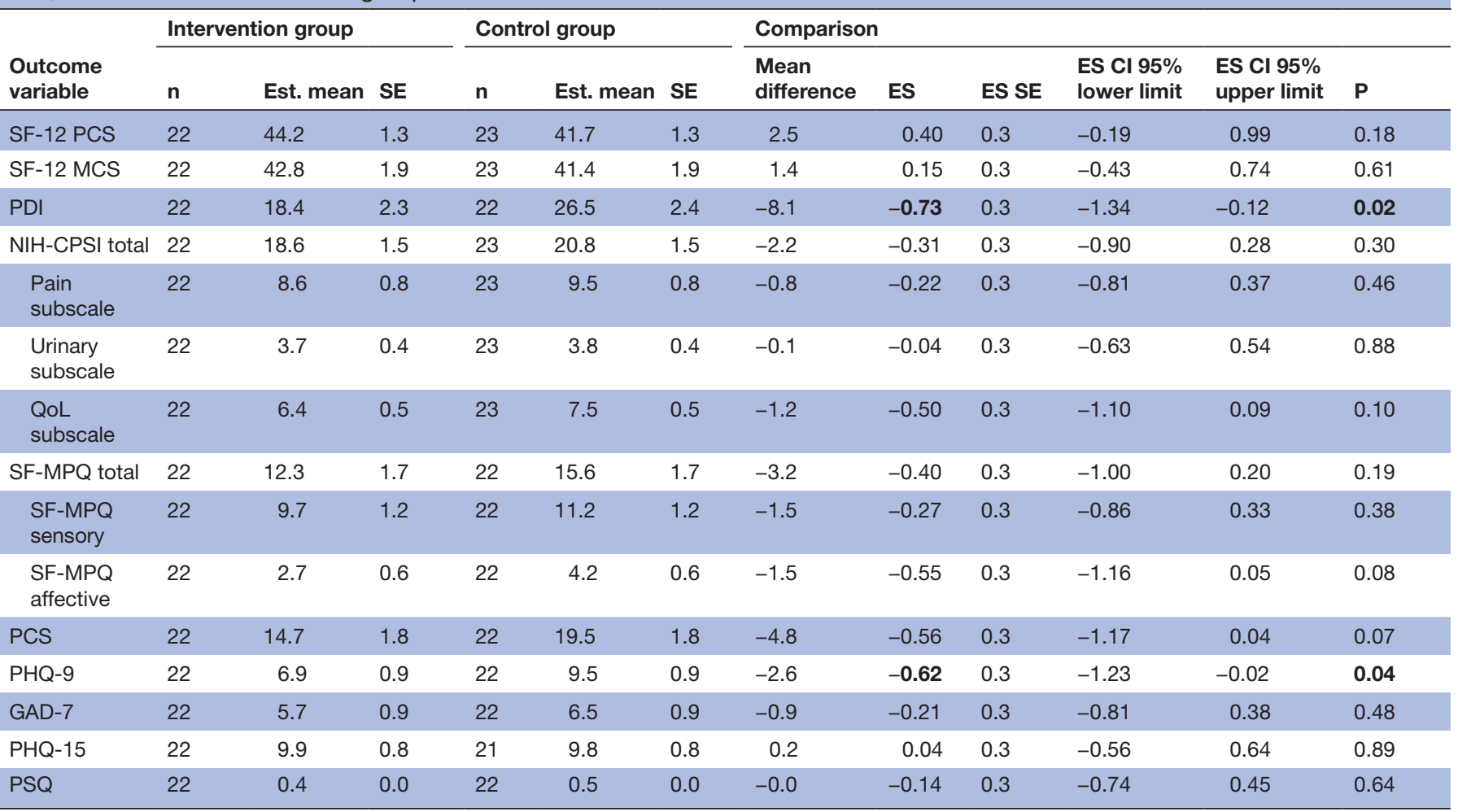

$P$ values $<0.05$ and corresponding ES are presented in bold.

ES, effect size Cohens' d; ES Cl, Cl interval of the effect size; ES SE, SE error of the effect size; Est., estimated; GAD-7, Patient Health Questionnaire Generalized Anxiety Disorder Screener; NIH-CPSI, National Institutes of Health Chronic Prostatitis Symptom Index; PCS, Pain Catastrophizing Scale; PDI, Pain Disability Index; PHQ-9, Patient Health Questionnaire 9 (depressive symptoms); PHQ-15, Patient Health Questionnaire 15 (severity of somatic symptoms); PSQ, Perceived Stress Questionnaire; QoL, Quality of Life; SF-12 MCS, 12-Item Short-Form Health Survey Mental Component Summary; SF-MPQ, Short-Form McGill Pain Questionnaire; SF-MPQ affective, affective subscale of the Short-Form McGill Pain Questionnaire; SF-MPQ sensory, sensory subscale of the Short-Form McGill Pain Questionnaire; SF-12 PCS, 12-Item Short-Form Health Survey Physical Component Summary. 


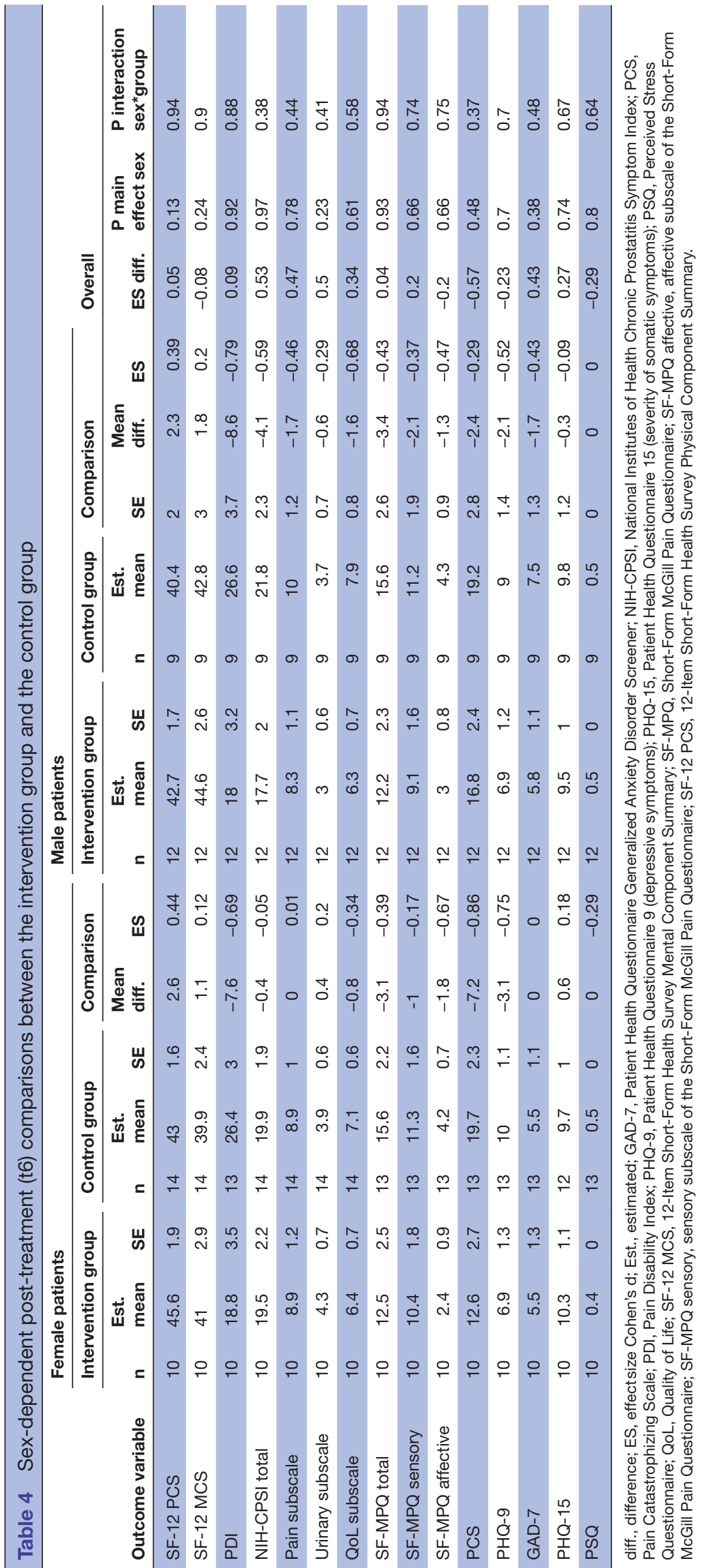




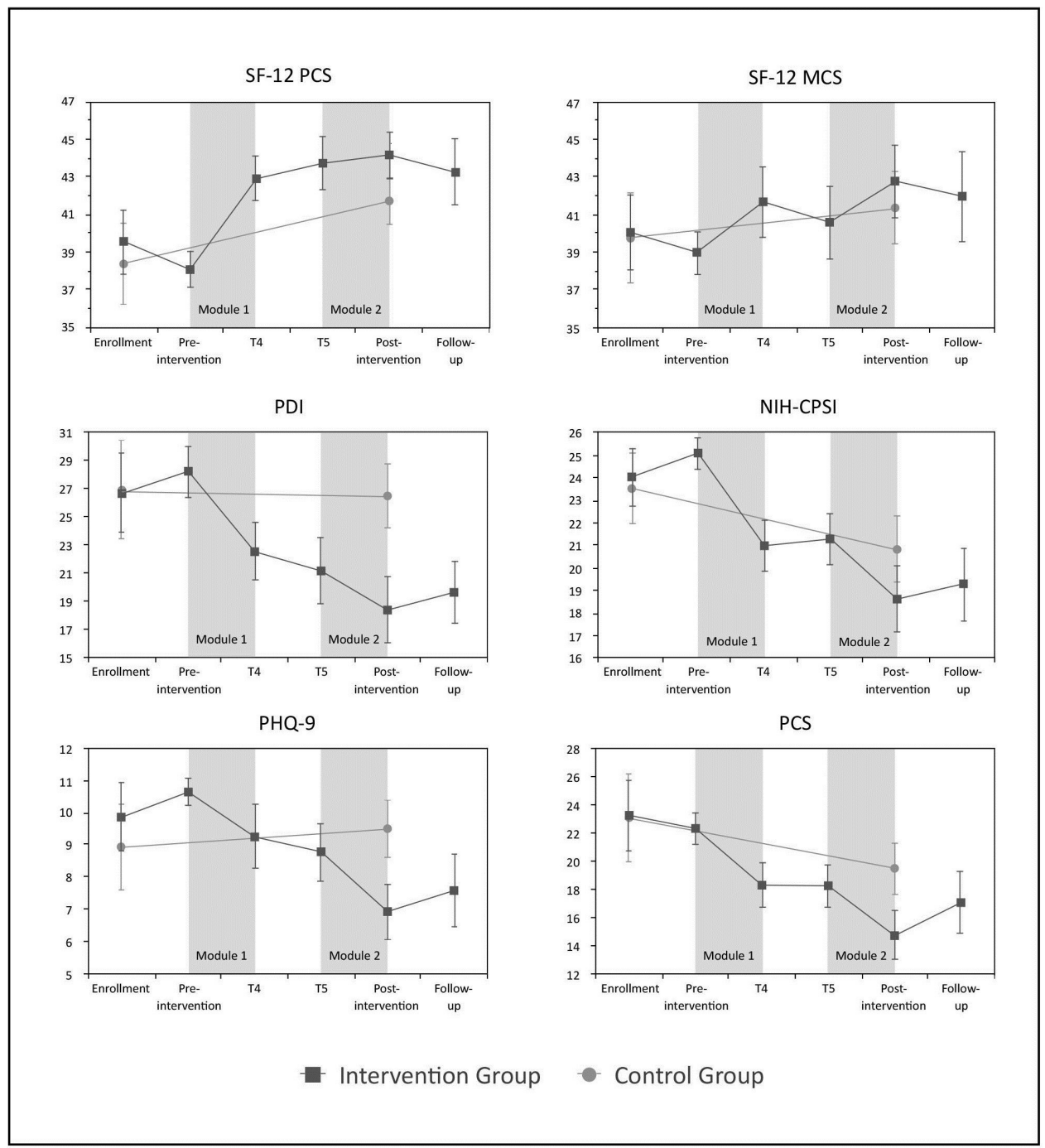

Figure 2 Course of important outcome variables in the intervention and the control group. SF-12 PCS, 12-Item Short-Form Health Survey Physical Component Summary; SF-12 MCS, 12-Item Short-Form Health Survey Mental Component Summary; PDI, Pain Disability Index; NIH-CPSI, National Institutes of Health Chronic Prostatitis Symptom Index; PHQ-9, Patient Health Questionnaire 9; PCS, Pain Catastrophizing Scale.

of delivering and evaluating. Although several challenges arose during recruitment, the intended sample size could be reached and participants expressed high satisfaction with the treatment. Furthermore, we received some insights on possible treatment effects in comparison with the treatment-as-usual group. Specifically, we found significant lower symptom burden in the intervention group as measured with the PDI and the PHQ-9 but no significant changes in the SF-12. Our results showed that delivering a combination of psychotherapy and physiotherapy was feasible; however, based on experiences in this study, some adaptations when conducting this programme in the future seem necessary. The evaluation of this intervention also demonstrated to be feasible using analysis of covariances; however, some instruments seemed to be more suitable in demonstrating effects than others.

Compared with the literature, ${ }^{46}$ the eligibility rate and the willingness-to-participate rate were lower than the median rates in other clinical trials. One of the main reasons of the low eligibility was the circumstance that patients could refer themselves to the specialised outpatient clinic. Thus, many patients did not have a CPPS diagnosis or were only interested in the diagnostic algorithm but not in the treatment study. Moreover, the low eligibility rate might be attributed to the time lag between initial eligibility screening and trial inclusion. In our study, up to $31 / 2$ years have passed since the patient's last appointment at the outpatient clinic and the inquiry for the study. Since it was a rather long time, several factors might have affected eligibility: First, many patients were unattainable due to relocations or other, mostly unknown, reasons. Second, given the natural course of chronic pain, nearly one-third of the patients have less symptoms over time or are even symptom free. ${ }^{47}$ Third, patients with CPPS were likely to use other healthcare services in order to find pain relief. ${ }^{48}$ Future trials should strive for a shorter time period between first contact with the patient and trial inclusion. Nevertheless, although the 
recruitment process faced these challenges, the intended sample size could be reached underlining the feasibility of the study. The feasibility of the physiotherapy and psychotherapy combination treatment was also supported by the low dropout rates for the intervention in total and for psychotherapy and physiotherapy separately. These rates were smaller in comparison to the literature ${ }^{49} 50$ and indicated high acceptance of the treatment. Finally, the feasibility is also indicated by the high level of satisfaction expressed by the participants. Satisfaction with the treatment is suggested to be a basic component for carrying out a successful psychotherapeutic and physiotherapeutic treatment. ${ }^{51}$ However, directly comparing this study with existing studies is difficult, since, to the best of our knowledge, this is the first study to investigate combined physiotherapy and psychotherapy in patients with CPPS.

While the eligibility rate was still within the IQR of examined studies by Gross et $a l^{46}{ }^{46}$ the willingnessto-participate rate was considerably below the IQR. Although the majority of persons perceived research to be very important, the willingness to participate often depends on convenience and whether or not study participation interfered with the daily routine. ${ }^{52}$ Moreover, patients are more likely take part in a study if the homestudy site distance is short. ${ }^{53}$ In our study, perceived lack of time, long distance to study site, and/or no interest were the most common reasons to refuse participation. Our willingness to participate rate would have improved substantial if we had delivered as least some parts of the intervention in a flexible, possible online format. Hence, these barriers should be targeted when designing future studies. One possible solution might be to concept at least some of the treatment sessions as online sessions. Not only do online programmes enable treatments independent of the home-study site distance, but also allow participants to better integrate the content of the therapy into their daily routine. ${ }^{54}$ Furthermore, online programmes provide continuity of care during pandemic situations such as the COVID-19 outbreak. ${ }^{55}$ Taking these adaptations in mind, we deem our combined intervention feasible and accepted by the patients.

Besides delivering feasibility, we also looked at effect sizes in order to explore evaluating feasibility. Several psychometric indicators showed that the intervention group improved in comparison to the control group although only the estimation of effect size measured with the PDI and the PHQ-9 reached significance level. Nevertheless, the intervention seems to be more effective than treatment as usual in terms of reduction of pain disabilities and depressive symptoms. Interestingly, the sequence psychotherapy first, physiotherapy second appears to be more effective than the other way around. Similar findings were observed in patients with chronic neck pain, who had greater effects in pain and disability reduction as well as quality of life when combining psychotherapy with subsequent physiotherapy. The authors conclude, that patients would need the physical performance in which they can apply and train the theoretical content of the
CBT. ${ }^{56}$ We have found that the intervention effects did not differ by gender. One possible explanation could be that women and men with CPPS have similar symptom patterns. Previous studies have shown that both sexes had similar pain intensity levels ${ }^{57}$ and that the proportion of mental disorders is elevated in comparison to the general population in both women and men. ${ }^{16}$ Hence, with the assumption of symptoms akin, the intervention might have had worked similar for female and male patients with CPPS. Nevertheless, the sex-disaggregated subsamples were small, which might affect the effect sizes. ${ }^{58}$

Prior to conducting an RCT, it is important to perform a power calculation to estimate the optimum sample size. For this purpose, the given effect sizes can be used. The COVID-19 pandemic also shows that online formats can be helpful to avoid treatment interruptions and to reach patients from rural areas more easily. An important point is that in addition to the professional groups involved, the patients' perspective should be included in the study design. While this feasibility study focused on acceptance, the next step should be to investigate the efficacy of the treatment with an appropriate design. Future studies should emphasise possible sex differences in order to tailor the interventions more specifically and effectively to the respective target group. To increase generalizability, a multicentre study would be the best option.

\section{Limitations}

Some limitations of the study should be mentioned. The SF-12 showed only a small and non-significant effect. The failure to detect a significant effect might be attributed to the small sample size of the study, but it could also be due to the generic nature of the instrument, which is not precise enough to detect changes in quality of life in patients with CPPS. This phenomenon was observed in patients with chronic low back pain ${ }^{59}$ and thus might also be true for patients with CPPS. Usage of a CPPS-specific instrument such as the NIH-CPSI ${ }^{36}$ instead of generic outcomes might be considered in future trials. Furthermore, this study is a feasibility study, which included a small, non-sufficient sample for testing the feasibility of the evaluation and for efficacy testing. Due to the small sample, we rather focused on the effect size Cohen's d than on the statistical significance. Although the effect size is more robust in small samples than the $p$ value, it is not completely unaffected by sample size..$^{58}$ Owing to the construction of the study as a monocentric pilot study, allocation to intervention and control group was non-randomised, which might cause variations in the distribution of sample characteristics. However, no significant differences in study characteristics could be detected between the two branches, which does not give support for the presence of bias. Thus, at this stage of research a non-randomised feasibility study seemed reasonable. It provides first hints that a combined physiotherapy and psychotherapy treatment might be beneficial and that the evaluation of the effect using psychometric questionnaires focussing on pain disabilities rather than 
quality of life is feasible. However, some studies, which administered either physiotherapy or psychotherapy, exist. The German concept reflective respiratory physiotherapy as such has not been tested, but the American Wise-Anderson Protocol includes similar therapeutic elements. A case series with male patients demonstrated decreased pain intensity and improved quality of life. ${ }^{60}$ The psychotherapeutic programme applied in this study was tested with a group of Canadian men showing positive effects in terms of pain intensity, catastrophising and quality of life ${ }^{61}$ In comparison, the combination of both therapeutic approaches in this study also indicate, among other positive effects, that pain and catastrophising decreased, and quality of life increased. Nonetheless, since existing studies are highly heterogeneous, comparing this study with the available literature should be viewed with caution. Furthermore, the absence of a patient perspective in the design of the study may also have an impact on the acceptance of the therapy.

Finally, we would like to state that this study provides valuable insights for further randomised, multicentre studies; not only regarding the acceptance and the effect of the intervention, but also regarding the recruitment process. The first results of a combined physiotherapeutic and psychotherapeutic treatment for patients with CPPS appear to be promising although some adaptations to the treatment programme had to be made as outlined above. Further testing of this procedure is therefore urgently needed to provide adequate and scientifically based treatment for patients with CPPS.

\section{Author affiliations \\ 1Department of Psychosomatic Medicine and Psychotherapy, University Medical Center Hamburg-Eppendorf, Hamburg, Germany \\ ${ }^{2}$ Department of Urology, University Medical Center Hamburg-Eppendorf, Hamburg, Germany \\ ${ }^{3}$ Department of Physiotherapy, University Medical Center Hamburg-Eppendorf, Hamburg, Germany \\ ${ }^{4}$ Department of Gynaecology, University Medical Center Hamburg-Eppendorf, Hamburg, Germany}

Acknowledgements The authors would like to thank the physiotherapists who were involved in this study, Gabriele Härtig, Ines Sallach, Matthias Bolik and Wilfried Evers as well as the psychotherapists Sonja Gregorzik, Katinka Kurz and Sebastian Schmidt. We also thank Yiqi Pan for her perceptive English editing services. Furthermore, we are grateful to Dean Tripp for giving us the opportunity to build on his previous work with patients with chronic pelvic pain syndrome and allowing us to adapt his patient workbook. Finally, we thank all participants who contributed to this study.

Contributors CAB: conceptualization, writing—review \& editing, supervision, project administration, funding acquisition. SGRK: investigation, data curation, writing —original draft, visualization. CD: formal analysis, investigation, data curation, writing —review \& editing, visualization; RA: investigation, writing —review \& editing; JH: resources, writing — review \& editing; MF: resources, writing—review \& editing; GK: conceptualization, writing — review \& editing, funding acquisition; BL: guarantor, conceptualization, resources, writing—review \& editing, supervision, funding acquisition.

Funding This work was supported by the PRANA Foundation in the Stifterverband für die Deutsche Wissenschaft e.V. (grant number: not applicable). The funders played no role in the design, conduct or reporting of this study.

Competing interests GK declares that she is a co-founder of the Association for Reflective Respiratory Physiotherapy (Verein für Reflektorische Atemtherapie e.V.), which was established in 2000. She has been a freelance lecturer for reflective respiratory physiotherapy for over 15 years. The other authors declare that they have no competing interests.

Patient consent for publication Not applicable.

Ethics approval This study involves human participants and was approved by Ethics Committee of the Medical Association Hamburg, Germany (reference number PV4220 for the outpatient clinic and reference number PV4801 for the feasibility study). Participants gave informed consent to participate in the study before taking part.

Provenance and peer review Not commissioned; externally peer reviewed.

Data availability statement Data are available upon reasonable request. Technical appendix, statistical code, and dataset available upon reasonable request from the corresponding author.

Supplemental material This content has been supplied by the author(s). It has not been vetted by BMJ Publishing Group Limited (BMJ) and may not have been peer-reviewed. Any opinions or recommendations discussed are solely those of the author(s) and are not endorsed by BMJ. BMJ disclaims all liability and responsibility arising from any reliance placed on the content. Where the content includes any translated material, BMJ does not warrant the accuracy and reliability of the translations (including but not limited to local regulations, clinical guidelines, terminology, drug names and drug dosages), and is not responsible for any error and/or omissions arising from translation and adaptation or otherwise.

Open access This is an open access article distributed in accordance with the Creative Commons Attribution Non Commercial (CC BY-NC 4.0) license, which permits others to distribute, remix, adapt, build upon this work non-commercially, and license their derivative works on different terms, provided the original work is properly cited, appropriate credit is given, any changes made indicated, and the use is non-commercial. See: http://creativecommons.org/licenses/by-nc/4.0/.

\section{ORCID iDs}

Susanne G R Klotz http://orcid.org/0000-0002-9886-9032

Bernd Löwe http://orcid.org/0000-0003-4220-3378

\section{REFERENCES}

1 Engeler D, Baranowski A, Berghmans B. Guidelines on chronic pelvic pain. Arnhem: European Association of Urology, 2019.

2 Ahangari A. Prevalence of chronic pelvic pain among women: an updated review. Pain Physician 2014;17:E141-7.

3 Grace VM, Zondervan KT. Chronic pelvic pain in New Zealand: prevalence, pain severity, diagnoses and use of the health services. Aust N Z J Public Health 2004;28:369-75.

4 Krieger JN, Lee SWH, Jeon J, et al. Epidemiology of prostatitis. Int J Antimicrob Agents 2008;31:85-90.

5 Pitts M, Ferris J, Smith A, et al. Prevalence and correlates of three types of pelvic pain in a nationally representative sample of Australian men. J Sex Med 2008;5:1223-9.

6 Díaz-Mohedo E, Hita-Contreras F, Luque-Suárez A, et al. Prevalence and risk factors of pelvic pain. Actas Urol Esp 2014;38:298-303.

7 Doiron RC, Tripp DA, Tolls V, et al. The evolving clinical picture of chronic prostatitis/chronic pelvic pain syndrome (CP/CPPS): a look at 1310 patients over 16 years. Can Urol Assoc J 2018;12:196-202.

8 Magistro G, Wagenlehner FME, Grabe M, et al. Contemporary management of chronic prostatitis/chronic pelvic pain syndrome. Eur Urol 2016;69:286-97.

9 Cohen JM, Fagin AP, Hariton E, et al. Therapeutic intervention for chronic prostatitis/chronic pelvic pain syndrome (CP/CPPS): a systematic review and meta-analysis. PLoS One 2012;7:e41941.

10 Champaneria R, Daniels JP, Raza A, et al. Psychological therapies for chronic pelvic pain: systematic review of randomized controlled trials. Acta Obstet Gynecol Scand 2012;91:281-6.

11 Klotz SGR, Schön M, Ketels G, et al. Physiotherapy management of patients with chronic pelvic pain (CPP): a systematic review. Physiother Theory Pract 2019;35:516-32.

12 Shoskes DA, Nickel JC, Kattan MW. Phenotypically directed multimodal therapy for chronic prostatitis/chronic pelvic pain syndrome: a prospective study using UPOINT. Urology 2010;75:1249-53.

13 Baranowski AP. Chronic pelvic pain. Best Pract Res Clin Gastroenterol 2009;23:593-610.

14 Klotz SGR, Ketels G, Löwe B, et al. Myofascial findings and psychopathological factors in patients with chronic pelvic pain syndrome. Pain Med 2020;21:e34-44. 
15 Albrecht R, Löwe B, A Brünahl C, et al. [Chronic pelvic pain syndrome and personality-association of somatic symptoms and psychic structure]. Psychother Psychosom Med Psychol 2015;65:418-25.

16 Brünahl C, Dybowski C, Albrecht R, et al. Mental disorders in patients with chronic pelvic pain syndrome (CPPS). J Psychosom Res 2017;98:19-26.

17 Brünahl CA, Riegel B, Höink J, et al. [Psychosomatic aspects of chronic pelvic pain syndrome. Psychometric results from the pilot phase of an interdisciplinary outpatient clinic]. Schmerz 2014;28:311-8

18 Riegel B, Albrecht R, Ketels G, et al. Symptomschwere und Belastungsfaktoren bei Patienten mit einem chronischen Unterbauchschmerzsyndrom - Implikationen für einen interdisziplinären und multimodalen Therapieansatz. Entspannungsverfahren 2014;31:40-57.

19 Riegel B, Bruenahl CA, Ahyai S, et al. Assessing psychological factors, social aspects and psychiatric co-morbidity associated with chronic prostatitis/chronic pelvic pain syndrome (CP/CPPS) in men a systematic review. J Psychosom Res 2014;77:333-50.

20 Dybowski C, Löwe B, Brünahl C. Predictors of pain, urinary symptoms and quality of life in patients with chronic pelvic pain syndrome (CPPs): a prospective 12-month follow-up study. J Psychosom Res 2018;112:99-106.

21 Relton C, Torgerson D, O'Cathain A, et al. Rethinking pragmatic randomised controlled trials: introducing the "cohort multiple randomised controlled trial" design. BMJ 2010;340:c1066.

22 Klotz SGR, Ketels G, Richardsen B, et al. [Physiotherapeutic assessment of chronic pelvic pain syndrome : Development of a standardized physiotherapeutic assessment instrument for interprofessional cooperation in patients with chronic pelvic pain syndrome]. Schmerz 2018;32:188-94.

23 Brünahl CA, Klotz SGR, Dybowski C, et al. Combined cognitivebehavioural and physiotherapeutic therapy for patients with chronic pelvic pain syndrome (COMBI-CPPS): study protocol for a controlled feasibility trial. Trials 2018;19:20.

24 Piontek K, Ketels G, Albrecht R, et al. Somatic and psychosocial determinants of symptom severity and quality of life in male and female patients with chronic pelvic pain syndrome. J Psychosom Res 2019;120:1-7.

25 Merskey H, Bogduk N, International Association for the Study of Pain (IASP). Classification of chronic pain. Descriptions of chronic pain syndromes and definitions of pain terms. 2 nd edn. Seattle: International Association for the Study of Pain, 2002.

26 Brüne L. Reflektorische atemtherapie. 3th edn. Stuttgart, New York: Georg Thieme Verlag, 1994.

27 Jacobson E. You must relax: practical methods for reducing the tensions of modern living. 5th edn. New York: McGraw-Hill, 1978.

28 Tripp DA, Nickel JC. Live a better life in spite of chronic pelvic pain. The cognitive-behavioural symptom management program for chronic pelvic pain. Patient workbook. 1st edn. Ontario, 2007.

29 Ware J, Kosinski M, Keller SD. A 12-item short-form health survey: construction of scales and preliminary tests of reliability and validity. Med Care 1996;34:220-33.

30 Fliege H, Rose M, Arck P, et al. Validierung des "perceived stress questionnaire" (PSQ) an einer deutschen stichprobe. Diagnostica 2001;47:142-52.

31 Levenstein S, Prantera C, Varvo V, et al. Development of the perceived stress questionnaire: a new tool for psychosomatic research. J Psychosom Res 1993;37:19-32.

32 Kroenke K, Spitzer RL, Williams JBW. The PHQ-15: validity of a new measure for evaluating the severity of somatic symptoms. Psychosom Med 2002;64:258-66.

33 Löwe B, Decker O, Müller S, et al. Validation and standardization of the generalized anxiety disorder screener (GAD-7) in the general population. Med Care 2008;46:266-74.

34 Löwe B, Kroenke K, Herzog W, et al. Measuring depression outcome with a brief self-report instrument: sensitivity to change of the patient health questionnaire (PHQ-9). J Affect Disord 2004;81:61-6.

35 Schneider H, Brähler E, Ludwig M, et al. Two-year experience with the german-translated version of the NIH-CPSI in patients with $\mathrm{CP} /$ CPPS. Urology 2004;63:1027-30.

36 Litwin MS, McNaughton-Collins M, Fowler FJ, et al. The National Institutes of health chronic prostatitis symptom index: development and validation of a new outcome measure. chronic prostatitis Collaborative research network. J Urol 1999;162:369-75.
37 Clemens JQ, Calhoun EA, Litwin MS, et al. Validation of a modified National Institutes of Health chronic prostatitis symptom index to assess genitourinary pain in both men and women. Urology 2009;74:983-7.

38 Dillmann U, Nilges $\mathrm{P}$, Saile $\mathrm{H}$, et al. [Assessing disability in chronic pain patients.]. Schmerz 1994;8:100-10.

39 Pollard CA. Preliminary validity study of the pain disability index. Percept Mot Skills 1984;59:974.

40 Meyer K, Sprott H, Mannion AF. Cross-cultural adaptation, reliability, and validity of the German version of the pain catastrophizing scale. J Psychosom Res 2008;64:469-78.

41 Sullivan MJL, Bishop SR, Pivik J. The pain catastrophizing scale: development and validation. Psychol Assess 1995;7:524-32.

42 Tal A. Schmerzen evaluieren. Physiopraxis 2008;6:38-9.

43 Melzack R. The short-form McGill pain questionnaire. Pain 1987;30:191-7.

44 Dempster AP, Laird NM, Rubin DB. Maximum Likelihood from Incomplete Data via the EM Algorithm. Journal of the Royal Statistical Society. Series B 1977;39:1-38.

45 Cohen J. Statistical power analysis for the behavioral sciences. 2nd edn. Hillsdale, NJ: Lawrence Earlbaum Associates, 1988.

46 Gross CP, Mallory R, Heiat A, et al. Reporting the recruitment process in clinical trials: who are these patients and how did they get there? Ann Intern Med 2002;137:10-16.

47 Landmark T, Dale O, Romundstad P, et al. Development and course of chronic pain over 4 years in the general population: The HUNT pain study. Eur J Pain 2018;22:1606-16.

48 Clemens JQ, Stephens-Shields A, Naliboff BD, et al. Correlates of health care seeking activities in patients with urological chronic pelvic pain syndromes: findings from the MAPP cohort. $J$ Urol 2018;200:136-40.

49 Bados A, Balaguer G, Saldaña C. The efficacy of cognitivebehavioral therapy and the problem of drop-out. J Clin Psychol 2007;63:585-92.

50 Jannenga $\mathrm{H}$. The state of rehab therapy 2018, 2018. Available: http:// www2.webpt.com/e/8532/he-state-of-rehab-therapy-2018/5t8v45/ 791022737

51 Anhang Price R, Elliott MN, Zaslavsky AM, et al. Examining the role of patient experience surveys in measuring health care quality. Med Care Res Rev 2014;71:522-54.

52 Anderson A, Borfitz D, Getz K. Global public attitudes about clinical research and patient experiences with clinical trials. JAMA Netw Open 2018;1:e182969.

53 Schweitzer A, Akmatov MK, Kindler F, et al. The impact of distance and duration of travel on participation rates and participants' satisfaction: results from a pilot study at one study centre in pretest 2 of the German National Cohort. BMJ Open 2015;5:e007461.

54 Camerini L, Camerini A-L, Schulz PJ. Do participation and personalization matter? A model-driven evaluation of an Internetbased patient education intervention for fibromyalgia patients. Patient Educ Couns 2013;92:229-34.

55 Eccleston C, Blyth FM, Dear BF, et al. Managing patients with chronic pain during the COVID-19 outbreak: considerations for the rapid introduction of remotely supported (eHealth) pain management services. Pain 2020;161:889-93.

56 Monticone M, Ambrosini E, Rocca B, et al. Group-based multimodal exercises integrated with cognitive-behavioural therapy improve disability, pain and quality of life of subjects with chronic neck pain: a randomized controlled trial with one-year follow-up. Clin Rehabil 2017;31:742-52.

57 Clemens JQ, Clauw DJ, Kreder K, et al. Comparison of baseline urological symptoms in men and women in the MAPP research cohort. J Urol 2015;193:1554-8.

58 Fan X, Konold T. Statistical significance versus effect size. In: Peterson P, Baker E, McGaw B, eds. International encyclopedia of education. 3rd edn. Oxford: Elsevier, 2010: 444-50.

59 DeVine J, Norvell DC, Ecker E, et al. Evaluating the correlation and responsiveness of patient-reported pain with function and quality-oflife outcomes after spine surgery. Spine 2011;36:S69-74.

60 Anderson RU, Wise D, Sawyer T, et al. Integration of myofascial trigger point release and paradoxical relaxation training treatment of chronic pelvic pain in men. J Urol 2005;174:155-60.

61 Tripp DA, Nickel JC, Katz L. A feasibility trial of a cognitivebehavioural symptom management program for chronic pelvic pain for men with refractory chronic prostatitis/chronic pelvic pain syndrome. Can Urol Assoc J 2011;5:328-32. 